\title{
Epidemiology of Intestinal Polyparasitism among Schoolchildren In Sohag, Egypt
}

\author{
Nada A. El-Nadi, Eman K. Omran, Noha S. Ahmed, Eman F. Fadel \\ Department of Medical Parasitology, Faculty of Medicine, Sohag University, Egypt. \\ Correspondence to DrNoha S. Ahmed, Department of Medical Parasitology, Faculty of \\ Medicine, Sohag University, Sohag 2630, Egypt \\ E-mail: nohasammer@yahoo.com
}

\begin{abstract}
Introduction: Intestinal parasitic infections (IPI) are still public health problems in many communities, particularly among children in developing countries.This crosssectional study aimed to investigate the current prevalence and risk factors associated with intestinal polyparasitism (the concurrent infection with multiple intestinal parasite species) among schoolchildren in Sohag governorate, Egypt.

Methodology / Principal findings: Fecal samples were collected from 200 schoolchildren $(\mathbf{5 1 . 5 \%}$ boys and $\mathbf{4 8 . 5 \%}$ girls), preserved and examined by using formalin-ethyl acetate concentration technique and modified Kinyoun acid fast stain. Demographic information was collected by using a standardized questionnaire. Overall, 63.5\% of the children were found to be positive for at least one parasite species. Of these, $\mathbf{2 3 . 5 \%}$ were polyparasitized. The overall prevalence of Cryptosporidium, Giardia duodenalis, Entamebahistolytical dispar, Blastocystisinfections were $34 \%, 14.5 \%$ and $13 \%$, respectively. Univariate and multivariable logistic regression models showed that none of the age, gender, residence or family size were risk factors for polyparasitism.

Conclusions/Significance: Intestinal polyparasitism is prevalent among children in Sohag, Egypt. Hence, effective and sustainable control measures, including schoolbased periodic chemotherapy, providing adequate health education focused on good personal hygiene practices and proper sanitation, as well as safe drinking water supply should be implemented to reduce the prevalence and consequences of these infections in this population.
\end{abstract}

Keywords: intestinal parasitic infections; polyparasitism; schoolchildren.

\section{Introduction}

IPIs are among the most prevalent of human infections worldwide, causing significant morbidity and mortality. More than 3.5 billion people are affected, the majorities being children and have been termed as cancers of the developing nations $[\mathbf{1 , 2}]$.

Over 600 million school-aged children live in areas where these parasites are intensively transmitted, and are in need of treatment and preventive interventions $[\mathbf{3 , 4}]$. The lack of access to safe water, sanitation, and hygiene are the key factors for the high intensity of intestinal parasites that, in children, frequently have the clinical expression of malabsorption syndrome and gastrointestinal morbidity [5].

The feco-oral route is the most significant in the transmission of IPIs to humans. It can occur anthroponotically, zoonotically, foodborne or waterborne. When the water/soil is contaminated, the resilient infective forms (eggs, cysts or spores) of the pathogenic organisms can be transported to vegetables, fruit, hands, tools, handles doors, currency, etc. and be easily swallowed accidentally by humans. They can be transmitted also by flies [6]. 
Negative effects of helminth infections include diminished physical fitness and growth retardation, and delayed intellectual development and cognition [7].

In particular, intestinal protozoans, such as Cryptosporidium spp., $G$. intestinalis and E. histolytica are major causes of diarrhea in children [8,9].

Recent studies support the pathogenic nature of Blastocystis. It is now accepted that the classic clinical features of infection with this parasite include gastrointestinal symptoms such as nausea, anorexia, flatulence, and acute or chronic diarrhea [9].

There is a general acceptance that severe IPIs are likely to result in failure to thrive and poor educational performance. Moreover, recent studies highlighted the impact of polyparasitism on the child's immunity and showed that polyparasitism is associated with higher mortality rates and may increase the susceptibility to other infections relative to infection with a single parasite [2].

\section{Subjects and methods \\ Ethics Statement}

This cross sectional study was conducted from January 2015 to December 2015 after being authorized by the scientific ethics committee of our institute.

Participation of schoolchildren was voluntary after explaining the aim of the study. Individual written informed consents were obtained from enrolled children or their guardians before data and sample collection with brief explanation of the procedure and the purpose of the study.

\section{Study Area}

Sohag is one of the governorates of upper Egypt. The capital, Sohag city, is located $467 \mathrm{~km}$ south Cairo. It is composed of $\mathbf{1 1}$ districts stretched from North to South along the Nile.

\section{Selection criteria}

200 school-aged children between 6 and 12 years, who attend primary schools in our governorate, were randomly recruited from four primary schools to be included in the study namely, Yousof Al-Kashif, Nag' Fadel, Arab Al Atawla\&Saqolta Al-Qadema Primary Schools.

\section{Questionnaire survey}

A standardized questionnaire was completed by children or their parents, who had given informed consent, in order to obtain a demographic description including the age, gender, residence, presence of symptoms and number of family members.

\section{Parasitological methods}

Stool specimens were collected in clean, dry, wide-mouth containers with tight-fitting lids. The acceptable amount of stool required for $\mathrm{O} / \mathrm{P}$ examination is 2 to $5 \mathrm{~g}$, often referred to as the size of a walnut.

The specimen container was labeled with the patient's name and identification number and the date and time of sample collection.

Specimens were immediately preserved after passage due to the lag time till they reach the laboratory. Preservatives used in this study were formalin $10 \%$ in 100 cases and sodium acetate-acetic acid-formalin (SAF) in the other $\mathbf{1 0 0 .}$

Microscopic examination was done after performing the formalin ethyl acetate concentration technique followed by staining with the kinyoun's acid fast stain for intestinal coccidia [3].

\section{Statistical analysis}

Results were gathered, organized and tabulated in an Excel 2010 spreadsheet. Data were analyzed using SPSS program for Windows version (22.0). Quantitative data were expressed as means \pm standard deviation. Qualitative data was expressed as frequencies and percentages. Chi-Square test $\left(\chi^{2}\right)$ and 
SOHAG MEDICAL JOURNAL

Vol. 21 No.1 Jan 2017

Fisher's Exact test were used when appropriate for comparison between qualitative variables. used in this study. Univariate and Multivariate logistic regression analysis were performed for identification of certain risk factors for polyparasitism. A $\mathbf{5 \%}$ level was chosen as a level of significance in all statistical tests.

Family size was categorized into two groups ( $<\mathbf{5}$ and $\geq \mathbf{5}$ members), and age of participants was categorized into two groups that were $<\mathbf{1 0}$ years and $\geq \mathbf{1 0}$ years according to previous studies.

\section{RESULTS}

\section{POPULATION PROFILE}

200 schoolchildren aged between 6 and 12 years (Age Mean \pm SD $=8.9 \pm 1.9$ ) had participated in this study.

Table 1. Demographics of the studied children $(n=200)$.

\begin{tabular}{|l|c|}
\hline Demographic characteristics & n (\%) \\
\hline Age (Mean \pm SD $=8.9 \pm 1.9)$ & $119(59.5)$ \\
\hline$<10$ years & $81(40.5)$ \\
\hline$\geq 10$ years & $97(48.5)$ \\
\hline Gender & $103(51.5)$ \\
\hline Girl & $100(50)$ \\
\hline Boy & $100(50)$ \\
\hline Residence & \\
\hline Urban & $42(21)$ \\
\hline Rural & $158(79)$ \\
\hline Family size (Mean \pm SD $=3.5 \pm 1.13)$ \\
\hline$<5$ members
\end{tabular}

Overall, $127(63.5 \%)$ of the children were found to be positive for at least one intestinal parasite species (Table 2).

Table 2. Infection prevalence among the studied children $(n=200)$.

\begin{tabular}{|l|c|c|}
\hline & $\mathbf{n}$ & $\%$ \\
\hline No. of parasitized children & $(127 / 200)$ & $63.5 \%$ \\
\hline No. of non-parasitized children & $(73 / 200)$ & $36.5 \%$ \\
\hline
\end{tabular}

Monoparasitized children constituted 80 (40\%) of children while (23.5\%) were polyparasitized. This is demonstrated in table $\mathbf{3}$. 
Table 3. Types of parasitism $(n=200)$.

\begin{tabular}{|c|c|c|}
\hline Parasitism & $\mathbf{n}$ & $(\%)$ \\
\hline Monoparasitism & $\mathbf{8 0}$ & $40 \%$ \\
\hline Polyparasitism* & 47 & $23.5 \%$ \\
\hline
\end{tabular}

In the current study, (23.5\%) of the studied children were polyparasitized. Out of them, $(\mathbf{1 7 . 5 \%})$ had pure protozoal co-infections and $(\mathbf{6 \%})$ were parasitized by protozoa and helminths simultaneously.

Table 4. Frequencies of protozoan and helminthic parasitisms $(n=200)$.

\begin{tabular}{|c|c|}
\hline & $\mathbf{n}(\%)$ \\
\hline Monoparasitism & $\mathbf{8 0}(40)$ \\
Helminths & $\mathbf{8}(4)$ \\
Protozoa & $72(36)$ \\
\hline Polyparasitism & $47(23.5)$ \\
Helminths+ Protozoa & $12(6)$ \\
Protozoa + Protozoa & $35(17.5)$ \\
\hline No parasitism $(\mathbf{n}=\mathbf{7 3})$ & $\mathbf{7 3}(36.5)$ \\
\hline
\end{tabular}

The prevalence of infections with pathogenic protozoa is shown in table 5 . Cryptosporidium was the most common pathogenic protozoan with a prevalence of 68(34\%) followed by $\boldsymbol{G}$. intestinalis $(\mathbf{1 4 . 5 \% )}, \boldsymbol{E}$. histolytical dispar(13\%), Blastocystis (10.5\%) and $\boldsymbol{C}$. cayetanensis $(\mathbf{6 . 5 \%})$ based on microscopy. 
Table 5.Parasite frequencies and percentages in descending manner.

\begin{tabular}{|c|c|}
\hline & $\mathrm{n}(\%)$ \\
\hline \multicolumn{2}{|l|}{ Protozoa } \\
\hline \multicolumn{2}{|l|}{ Pathogenic } \\
\hline Cryptosporidium & $68(34)$ \\
\hline G. intestinalis & $29(14.5)$ \\
\hline E. histolytica/ dispar & $26(13)$ \\
\hline Blastocystis & $21(10.5)$ \\
\hline C. caytanensis & $13(6.5)$ \\
\hline \multicolumn{2}{|l|}{ Non-pathogenic } \\
\hline E. coli & $7(3.5)$ \\
\hline I. butscilli & $5(2.5)$ \\
\hline C. mesnilli & $3(1.5)$ \\
\hline E. hartmanni & $3(1.5)$ \\
\hline \multicolumn{2}{|l|}{ Helminths } \\
\hline H. nana & $10(5)$ \\
\hline A. lumbricoides & $4(2)$ \\
\hline E. vermicularis & $4(2)$ \\
\hline A. duodenale & $3(1.5)$ \\
\hline
\end{tabular}

Results showed that children exhibited various forms of poly-parasitism. Twospecies infection was the most common (24.4\%) followed by Three-species infection (7.9\%), four-species infection (3.9\%) and lastly one child had five-species infection $(0.8 \%)$.

Table 6. Frequencies of different forms of polyparasitism (mixed protozoal\&helminth infections).

\begin{tabular}{|l|c|c|}
\hline & $\mathrm{n}$ & $\%$ \\
\hline Double infection & 31 & 24.4 \\
\hline Triple infection & 10 & 7.9 \\
\hline Quadruple infection & 5 & 3.9 \\
\hline Pentaple infection & 1 & 0.8 \\
\hline
\end{tabular}

Polyparasitism with two protozoan parasites were found in $\mathbf{( 1 7 . 3 \% )}$ of children. The most common dual infection was with E. histolytica/ disparandBlastocystis with a prevalence of $\mathbf{( 3 . 1 \% )}$. In addition, $\mathbf{( 2 . 4 \% )}$ of children exhibited triple infections withE. histolytica/ dispar, G. intestinalisandBlastocystis. Other cases of polyparasitism are shown in table 7. 
Table 7.Protozoal co-infections among parasitized children $(n=127)$.

\begin{tabular}{|c|c|}
\hline & $\mathrm{n}(\%)$ \\
\hline Double infection & \\
\hline E. histolytica/ dispar, Blastocystis & $4(3.1)$ \\
\hline Giardia, Blastocystis & $3(2.4)$ \\
\hline E. histolytica/ dispar, Giardia & $5(3.9)$ \\
\hline Giardia, C. mesnilli & $1(0.8)$ \\
\hline Giardia, E. coli & $5(3.9)$ \\
\hline Blastocystis, I. butscilli & $2(1.6)$ \\
\hline E. histolytica/ dispar, I. butscilli & $2(1.6)$ \\
\hline Total & $22(17.3)$ \\
\hline Triple infection & $3(2.4)$ \\
\hline E. histolytica/ dispar, Giardia, Blastocystis \\
\hline
\end{tabular}

Results revealed that the type of polyparasitzed children were more frequently symptomatized than monoparasitized children. A statistical significance was found between diarrhea and the type of parasitism. P-value was $\mathbf{0 . 0 1 2}$ as shown in table $\mathbf{8}$. Other symptoms showed no statistical significance.

Table 8. Relation between symptoms and type of parasitism amongst parasitized children $(n=127)$.

\begin{tabular}{|c|c|c|c|}
\hline & $\begin{array}{c}\text { Monoparasitism } \\
(\mathbf{N}=\mathbf{8 0})\end{array}$ & $\begin{array}{c}\text { Polyparasitism } \\
(\mathrm{N}=47)\end{array}$ & P-value \\
\hline $\begin{array}{c}\text { Diarrhea } \\
\text { Yes } \\
\text { No } \\
\end{array}$ & $\begin{array}{l}12(42.9 \%) \\
68(68.7 \%)\end{array}$ & $\begin{array}{l}16(57.1 \%) \\
31(31.3 \%)\end{array}$ & $0.012 *$ \\
\hline $\begin{array}{l}\text { Pain } \\
\text { Yes } \\
\text { No }\end{array}$ & $\begin{array}{c}6(42.9 \%) \\
74(65.5 \%)\end{array}$ & $\begin{array}{c}8(57.1 \%) \\
39(34.5 \%)\end{array}$ & 0.098 \\
\hline $\begin{array}{c}\text { Dysentery } \\
\text { Yes } \\
\text { No } \\
\end{array}$ & $\begin{array}{l}0(0.0 \%) \\
80(64 \%)\end{array}$ & $\begin{array}{l}2(100 \%) \\
45(36 \%)\end{array}$ & 0.135 \\
\hline $\begin{array}{c}\text { Perianal itching } \\
\text { Yes } \\
\text { No }\end{array}$ & $\begin{array}{c}2(66.7 \%) \\
78(62.9 \%)\end{array}$ & $\begin{array}{c}1(33.3 \%) \\
46(37.1 \%)\end{array}$ & 0.894 \\
\hline
\end{tabular}

P- value was calculated by Chi square test and Fisher's Exact Test *Statistically significant 


\section{RISK FACTORS FOR POLYPARASITISM}

Rates of polyparasitism were relatively lower than monoparasitism in both age groups. No statistical significance was denoted. $\mathrm{P}$ value was $\mathbf{0 . 4 2 5}$.

Boys and girls from both urban anr rural areas were more commonly monoparasitized. No statistical significance was found.

Also family size did not significantly affect the type of parasitism. $\mathrm{P}$ value was 0.295.

Table 9. Type of parasitism and demographic features among infected children (127).

\begin{tabular}{|c|c|c|c|}
\hline & $\begin{array}{c}\text { Monoparasitism } \\
(\mathbf{N}=\mathbf{8 0})\end{array}$ & $\begin{array}{c}\text { Polyparasitism } \\
(\mathrm{N}=47)\end{array}$ & P-value \\
\hline Age & & & \\
\hline$<10$ years & $50(65.8 \%)$ & $26(34.2 \%)$ & 0.425 \\
\hline$\geq 10$ years & $30(58.8 \%)$ & $21(41.2 \%)$ & \\
\hline Sex & & & 0.425 \\
\hline Boys & $45(66.2 \%)$ & $32(33.8 \%)$ & \\
\hline Girls & $35(59.3 \%)$ & $24(40.7 \%)$ & \\
\hline Residence & & & 0.919 \\
\hline Urban & $35(62.5 \%)$ & $21(37.5 \%)$ & \\
\hline Rural & $45(63.4 \%)$ & $26(36.6 \%)$ & \\
\hline Family size & & & 0.295 \\
\hline$<5$ members & $14(73.7 \%)$ & $5(26.3 \%)$ & \\
\hline$\geq 5$ members & $66(61.1 \%)$ & $42(38.9 \%)$ & \\
\hline
\end{tabular}

P- value was calculated by Chi square test

*Statistically significant

Univariate and multivariable logistic regression models were performed to identify risk factors that were expected to be significantly associated with intestinal polyparasitism. According to our data, none of the age, gender, residence or family size were risk factors for polyparasitism. See tables $\mathbf{1 0} \& \mathbf{1 1}$.

Table 10.Univariate logistic regression analysis of factors associated with polyparasitism.

\begin{tabular}{|r|c|c|}
\hline & OR $\left(\right.$ CI $\left.{ }_{95 \%}\right)$ & P - value \\
\hline Age & $0.7(0.4-1.5)$ & 0.426 \\
\hline Sex & $0.7(0.3-1.5)$ & 0.425 \\
\hline Residence & $0.9(0.5-1.9)$ & 0.919 \\
\hline Family size & $1.8(0.6-5.3)$ & 0.3 \\
\hline
\end{tabular}

OR, Odds ratio.CI, Confidence interval.

Significant association (unadjusted P,0.05). 
Table 11. Multivariate logistic regression analysis of factors associated with polyparasitism.

\section{Discussion}

\begin{tabular}{|l|c|c|}
\hline & Adjusted OR (CI 95\%) & P - value \\
\hline Age & $0.8(0.4-1.7)$ & 0.579 \\
\hline Sex & $0.8(0.4-1.6)$ & 0.456 \\
\hline Residence & $1.03(0.5-2.2)$ & 0.936 \\
\hline Family size & $1.7(0.5-5.1)$ & 0.374 \\
\hline
\end{tabular}

OR, Odds ratio.CI, Confidence interval. Significant association $(\mathbf{P , 0 . 0 5})$.

IPIs are still public health problems in many communities, particularly among children in developing countries. IPI are associated with high morbidity particularly among schoolchildren [2]. This study attempted to assess the prevalence of intestinal polyparasitism, and its associated risk factors.

The findings of the present crosssectional survey conducted in four randomly chosen governmental Primary schools in urban and rural Sohag governorate, Egypt showed that $\mathbf{( 6 3 . 5 \% )}$ of the participating children harboured at least one parsite species.

The almost non-changing situation of IPIs in Sohag is evident from the agreement of the present finding with that ofHamedet al., 2013 who reported the infection of $\mathbf{( 6 3 . 3 1 \% )}$ of rural children aged less than 12 in rural Sohag [11].

In contrast, a lower prevalence rate of $\mathbf{( 3 8 . 5 \% )}$ has been previously reported for IPIs among primary schoolchildren in Sohag [12]. Direct smear examination and FECT were used for fecal examination, scotch tape for $\boldsymbol{E}$. vermicularisand urine sample examination for $S$. hematobium but they did not perform any special staining procedures to detect Cryptosporidium spp.

However, Osman et al., (2016) recorded an overall prevalence of $\mathbf{( 8 5 \% )}$ ) among schoolchildren in Tripoli, Lebanon. This was explained by performing fecal examination by both microscopy and molecular tools in their study [10].
In this study,a predominance of protozoal compared to helminthic infections $\mathbf{( 5 3 . 5 \%}$ vs. 4\%) among schoolchildren was found. Such a prevalence is high relied on the collection of a single stool sample per child, instead of the ideal three consecutive samples.

Monoparasitism was the most predominant type of infection, being prevalent among $\mathbf{( 4 0 \% )}$ ) of schoolchildren, while only $\mathbf{( 2 3 . 5 \% )}$ ) were harboring double and triple infections. Our results are consistent with the results of acrosssectional survey which was carried out in earlier 2011 by Matthyset al. in western Tajikistan where $\mathbf{( 4 0 . 9 \% )}$ of all children had a single species infection, whereas 17.3\% had a dual species infection and 4.9\% harboured at least three intestinal pathogenic parasite species concurrently [8].

Co-infections were common, affecting $\mathbf{3 2 . 5 \% )}$ of schoolchildren in the Plateau Central and Centre-Ouest regions of Burkina Faso as reported by Erismannet al., (2017). This indicates that these environments are extremely contaminated [13].

Cryptosporidium (34\%) was strikingly found to be the highest prevalent parasite in this study, The major risk factor for cryptosporidium infection is drinking water that is contaminated with oocysts. Moreover, oocysts can survive in chlorine used for water treatment [14].

The most frequent protozoan detected in Central Maluku regency, Indonesia 
schoolchildren was Cryptosporidium sp. $\mathbf{( 2 4 . 7 \% )}$ [15]. The authors reported using the modified Ziehl-Neelsen staining technique.

Today, Blastocystis spp. is considered an under-reported parasite, with a worldwide distribution and a prevalence far exceeding that of other intestinal parasites in the human population. Indeed, its prevalence can reach $100 \%$ in developing countries and has been reported at between $\mathbf{1 . 5 \%}$ and $20 \%$ in industrialized countries [10].

Univariate and multivariate logistic regression models revealed that none of the age, gender, residence or family size were risk factors for polyparasitism. This is consistent with (Al-Delaimyet al., 2014) [2].

In the current study, (23.5\%) of study population were polyparasitized. Out of them, (17.5\%) had pure protozoalpolyparasitism and $\mathbf{( 6 \% )}$ were parasitized by protozoa and helminths simultaneously.

$22(\mathbf{1 7 . 3 \%})$ of the parasitized children had two species infections. The most common dual infection was with $E$. histolytical disparandBlastocystis spp. with a prevalence of $\mathbf{( 3 . 1 \% )}$. In addition, $(\mathbf{2 . 4 \%})$ of children exhibited triple infections with E. histolytical dispar, $G$. intestinalisandBlastocystis.

Jafariet al. (2014) stated that interestingly $E$. nana and $C$. mesnili were seen more in humans with loose feces, but we should note that some co-infections between these protozoans has been observed and consequently we don't know which one/ones were responsible for loose feces [16].

Interestingly, $\mathbf{( 5 . 4 \% )}$ of the polyparasitized children were infected by five different parasite species (mainly the three STH species, $\boldsymbol{G}$. duodenalis and $\boldsymbol{E}$. histolytica/ dispar) [2].

\section{STUDY LIMITATIONS}

First, the findings presented here cannot be generalized for all Sohag governorate schoolchildren. The random selection of schools with a sample size not large enough to be representative of the targeted community.

Second, this study was obliged to rely on a single fecal sample per child instead of the ideal three consecutive samples because of limitation of resources. Thus, the prevalence rate of parasitic infections is likely to be underestimated due to the temporal variation in egg excretion and cyst passage over hours and days.

A limitation of the present study was the use of infection prevalence but not infection intensity with STHs. Also, $S$. mansoni was never detected.

The shortcomings of this study perhaps made the rate of $E$. vermicularis infection clearly low among the most susceptible group, that is, schoolchildren.

Many villages with no road access and therefore were not covered by the current study. Thus, further investigations are necessary to more precisely evaluate the current status of the research problem.

Despite these limitations, our findings highlight a number of important issues. First, this study could be considered as a basis for conducting further studies that account for the determination of infection intensity, which could reflect the transmission dynamics of such parasites and their associated morbidity.

\section{Conclusion}

The findings of the present study revealed that IPIs are very common among schoolchildren in Sohag, Egypt. Intestinal polyparasitism is a significant problem among schoolchildren in Sohag, Egypt. Cryptosporidiosis was the most common infection detected. Hence, there is an urgent need to implement an innovative and integrated control program to reduce the prevalence and intensity of these infections significantly and to save these children from their negative impacts.

\section{References}

1. Tulu B., Taye S. \&Amsalu E. (2014). Prevalence and its associated risk factors of intestinal parasitic infections among Yadot primary school children of 
South Eastern Ethiopia: a crosssectional study. BMC Res. Notes, 7, 848.

2. Al-Delaimy A. K., Al-Mekhlafi H. M., Nasr N. A., Sady H., Atroosh W. M., Nashiry M., ... Mahmud R. (2014). Epidemiology of intestinal Polyparasitism among Orang Asli school children in rural Malaysia. PLoSNegl Trop Dis, 8(8), e3074.

3. Garcia L. S. (2016). Diagnostic medical Parasitology. ASM Press $6^{\text {th }}$ edition.

4. Opara K.N., Udoidung N.I., Opara D.C., Okon O.E., Edosomwan E.U., Udoh A.J. (2012). The Impact of Intestinal Parasitic Infections on the Nutritional Status of Rural and Urban School-Aged Children in Nigeria.Int $\mathbf{J}$ of MCH and AIDS, 1(1),73-82.

5. WHO (2016). Soil-transmitted helminth infections. Retrieved November 26, 2016, from World Health Organization, http://www.who.int/mediacentre/factshe ets/fs366/en/

6. Macchioni F., Segundo H., Gabrielli S., Totino V., Gonzales P. R., Salazar E., ... Cancrini G. (2015). Dramatic decrease in prevalence of soiltransmitted Helminths and new insights into intestinal protozoa in children living in the Chaco region, Bolivia. Am J Trop Med Hyg, 92(4), 794-796.

7. Lobo M. L., Augusto J., Antunes F., Ceita J., Xiao L., Codices V., \& Matos O. (2014). Cryptosporidium spp., Giardia duodenalis, Enterocytozoonbieneusi and other intestinal parasites in young children in Lobata province, democratic republic of São Tomé and Principe. PLoS ONE, 9(5), e97708.

8. Matthys B., Bobieva M., Karimova G., Mengliboeva Z., Jean-Richard V., Hoimnazarova M., ... Wyss K. (2011). Prevalence and risk factors of helminths and intestinal protozoa infections among children from primary schools in western Tajikistan. Parasit Vectors, 4(1), 195.
9. Mulatu G., Zeynudin A., Zemene E., Debalke S., \&Beyene G. (2015). Intestinal parasitic infections among children under five years of age presenting with diarrhoeal diseases to two public health facilities in Hawassa, south Ethiopia. Infect Dis Poverty, 4(1).

10. Osman M., El Safadi D., Cian A., Benamrouz S., Nourrisson C., Poirier, P., ... Certad G. (2016). Prevalence and risk factors for intestinal protozoan infections with Cryptosporidium, Giardia, Blastocystis and Dientamoebaamong schoolchildren in Tripoli, Lebanon. PLoSNegl Trop Dis, 10(3), e0004496.

11. Hamed A. F., Yousef F. M. A., Omran E. K. \&Moustafa A. (2013). Common Parasitic Infestation among Rural Population in Sohag Governorate, Egypt. J Am Sci; 9(4).

12. El-Masry H. M., Ahmed Y. A. A., Hassan A., Zaky S., Abd-Allah E. S., El-Moselhy E. A., Baraka Y. A. \& Abdel-RahemM. A.(2007). Prevalence, Risk Factors and Impacts of Schistosomal and Intestinal Parasitic Infections Among Rural School Children in Sohag Governorate. EJHM, 29: 616- 30

13. Erismann S., Knoblauch A. M., Diagbouga S., Odermatt P., Gerold J., Shrestha A., ... Cissé G. (2017). Prevalence and risk factors of undernutrition among schoolchildren in the plateau central and Centre-Ouest regions of Burkina Faso. Infect Dis Poverty, 6(1), 17.

14. Latif B. \&Rossle N. F. (2015). Cryptosporidiosis among children with diarrhoea in three Asian countries: A review. Asian Pacific Journal of Trop Biomed, 5(11), 885-888.

15. Sianturi M. D.G., Rahakbauw I. M., Meyanti F., Kusumasari A. R., Hartriyanti Y. E. \&Murhandarwati E. E. H. (2016). Prevalence of intestinal protozoan infections and association with hygiene knowledge among primary schoolchildren in Salahutu and Leihitu 
SOHAG MEDICAL JOURNAL

Vol. 21 No.1 Jan 2017

districts, Central Maluku regency,

Indonesia. Trop Biomed 33(3): 428-436.

16. Jafari R., Sharifi, F., Bagherpour B., \& Safari M. (2014). Prevalence of
Epidemiology of Intestinal Polyparasitism among

Eman Fathi Fadel

intestinal parasites in Isfahan city, central Iran, 2014. J Parasit Dis, 40(3), 679-682. 